\title{
Caracterização agronômica da produção de rizomas de clones de taro
}

\author{
Francisco Hevilásio F. Pereira ${ }^{1}$; Mário Puiatti ${ }^{1}$; Glauco V. Miranda ${ }^{1}$; Derly José H. da Silva ${ }^{1}$; Fernando \\ Luiz Finger ${ }^{1}$ \\ ${ }^{1 / U F V ~-~ D e p t o . ~ F i t o t e c n i a, ~ 36.571-000, ~ V i c ̧ o s a-M G . ~ E-m a i l: ~ m p u i a t t i @ u f v . b r ~}$
}

\begin{abstract}
RESUMO
Objetivou-se caracterizar agronomicamente 36 acessos (clones) de taro pertencentes ao Banco de Germoplasma de Hortaliças da UFV quanto às características relativas à produção de rizomas. $\mathrm{O}$ experimento foi conduzido na horta de pesquisas da UFV, de 19/09/ 2000 a 13/07/2001. Foi utilizado o delineamento de blocos casualizados, com 36 tratamentos (clones) e cinco repetições. A parcela foi composta de quatro fileiras, com quatro metros de comprimento, com as plantas espaçadas de 1,0 x 0,5 m. As características avaliadas foram peso médio de rizomas comerciáveis; número de rizomas comerciáveis por planta; produtividade de rizomas comerciáveis e total; razão de formato (diâmetro longitudinal/diâmetro transversal) de rizomas comerciáveis; produtividade de rizomas em classes (com base no diâmetro transversal) filho grande ( $>47 \mathrm{~mm}$ ), filho médio (40-47 mm), filho pequeno $(33-40 \mathrm{~mm}) \mathrm{e}$ refugo (<33 mm). Os clones BGH 5916, BGH 6137 e BGH 6298 apresentaram boa conformação, com a razão de formato entre $1,1 \mathrm{e}$ 1,7 (rizomas oblongos) e as melhores respostas agronômicas quanto ao peso médio de rizomas comerciáveis, produtividades comerciável e total, não diferindo do clone BGH 5925 (Japonês) e sendo maior que do clone BGH 5928 (Chinês), esses últimos os mais plantados no Brasil. Os clones BGH 6307 e BGH 6308, apesar de não apresentarem peso médio de rizomas comerciáveis tão elevado quanto os clones BGH 5916, BGH 6298 e BGH 6137, em razão do maior número de rizomas comerciáveis por planta, tiveram produtividades comerciáveis semelhantes a esses e boa produtividade total. Correlações positivas e significativas foram encontradas entre peso médio de rizomas comerciáveis $(0,6370$ e 0,7450), número de rizomas comerciáveis por planta $(0,7133$ e 0,5338$)$, produtividade de rizomas filho grande $(0,9107$ e 0,9451$)$ com as produtividades de rizomas comerciáveis e totais, respectivamente; entre a produtividade de rizomas comerciáveis $(0,9455)$ e produção de rizomas mãe $(0,8753)$ com a produtividade total; e entre peso médio de rizomas comerciáveis com produção de rizomas filho grande $(0,8519)$. Os componentes primários com maiores correlações positivas com a produtividade comerciável foram peso médio e número por planta de rizomas comerciáveis e produtividade de rizomas filho grande.
\end{abstract}

Palavras-chave: Colocasia esculenta (L.) Schott, inhame, clone, produtividade, peso médio de rizomas.

\begin{abstract}
Characterization of yield factors in taro clones

Clones of taro, from the Germoplasm Bank of the Universidade Federal de Viçosa, Brazil, were agronomically characterized. The experiment was carried out from 19/09/2000 to 13/07/2001, in a randomized complete block design, with 36 treatments (clones) and five replicates. The experimental unit was comprised of four rows spaced $1.0 \mathrm{~m}$ apart, with four meters in length. The distance between plants in the row was $0.5 \mathrm{~m}$. We evaluated the average commercial rhizome weight; number of commercial rhizomes/plant; total and commercial rhizome yield; format ratio (longitudinal/transversal diameter) of the commercial production, and rhizomes classification according to the transversal diameter (large offspring $>47 \mathrm{~mm}$; medium offspring $=40-47 \mathrm{~mm}$; small offspring $=33-40 \mathrm{~mm}$; and discard < 33 mm). Clones BGH 5916, BGH 6137, and BGH 6298 presented good rhizome shape (ratio between 1.1 and 1.7) and higher values of mean corm fresh commercial weight, commercial and total yields. These clones did not differ from BGH 5925 (Japanese), presenting significantly higher values than BGH 5928 (Chinese), the two most common taros grown in Brazil. Clones BGH 6307 and BGH 6308, presented slightly inferior commercial corm fresh weight than BGH 5916, BGH 6298, and BGH 6137. However, due to the higher commercial rhizomes number/plant, the commercial and total yields were similar. Correlation was positive and significant between average commercial corm fresh weight per plant (0.6370 and 0.7450$)$, number of commercial corm per plant (0.7133 and 0.5338 ), and yield of large corm offspring (0.9107 and 0.9451), when compared to commercial and total yields, respectively; between yield of commercial $(0.9455)$ and of mother corm $(0.8753)$ when compared to total yield; between commercial mean corm fresh weight (0.8519) when compared to yield of large offspring. Primary components presenting high positive correlation in comparison to commercial yield were: commercial mean corm fresh weight, number of commercial corm per plant and yield of large offspring.
\end{abstract}

Keywords: Colocasia esculenta (L.) Schott, dasheen, clone, yield, mean corm fresh weight.

\section{(Recebido para publicação em 14 de março de 2002 e aceito em 10 de outubro de 2002)}

$\mathrm{O}$ taro, Colocasia esculenta (L.) Schott, também conhecido como inhame no centro-sul do Brasil, ocupa lugar de destaque na agricultura e na dieta da população de muitos países tropicais e subtropicais. Os rizomas constituem-se em alimento amiláceo básico em diversas nações da Ásia, África e ilhas do Pacífico (Wang, 1983). O ami- do de rizomas de taro é conhecido por apresentar grânulos relativamente pequenos, quando comparado a outras amiláceas, além de suas qualidades químicas naturais, o que o torna uma rica fonte nutricional para humanos e animais, bem como um suprimento comercial para muitas aplicações industriais (Nip, 1990). Sua digestibilidade é ele- vada $(97 \%)$, proporcionando eficiente liberação dos componentes durante a digestão e absorção desse alimento (Standal, 1983).

A importância dos rizomas de taro não se limita apenas como fonte de carboidratos e proteínas, mas por suplementar, com vitaminas e sais minerais essenciais as dietas à base de cereais 
Tabela 1. Peso médio de rizomas filhos (PMR) de acessos de taro pertencentes ao BGH/UFV utilizados como material propagativo na instalação do experimento. Viçosa, UFV, 2000-2001.

\begin{tabular}{cccccccc}
\hline $\begin{array}{c}\text { Acesso } \\
(\text { BGH) }\end{array}$ & $\begin{array}{c}\text { PMR } \\
(\mathbf{g})\end{array}$ & $\begin{array}{c}\text { Acesso } \\
(\mathbf{B G H})\end{array}$ & $\begin{array}{c}\text { PMR } \\
(\mathbf{g})\end{array}$ & $\begin{array}{c}\text { Acesso } \\
(\mathbf{B G H})\end{array}$ & $\begin{array}{c}\text { PMR } \\
(\mathbf{g})\end{array}$ & $\begin{array}{c}\text { Acesso } \\
(\mathbf{B G H})\end{array}$ & $\begin{array}{c}\text { PMR } \\
(\mathbf{g})\end{array}$ \\
\hline BGH 5913 & 97 & BGH 5926 & 59 & BGH 6091 & 65 & BGH 6306 & 66 \\
BGH 5914 & 70 & BGH 5927 & 140 & BGH 6092 & 63 & BGH 6307 & 76 \\
BGH 5915 & 100 & BGH 5928 & 61 & BGH 6093 & 106 & BGH 6308 & 76 \\
BGH 5916 & 111 & BGH 5929 & 53 & BGH 6094 & 92 & BGH 6315 & 43 \\
BGH 5917 & 86 & BGH 5931 & 74 & BGH 6095 & 68 & BGH 6606 & 70 \\
BGH 5918 & 64 & BGH 6086 & 60 & BGH 6132 & 57 & BGH 6607 & 58 \\
BGH 5920 & 53 & BGH 6087 & 77 & BGH 6136 & 90 & BGH 6708 & 60 \\
BGH 5921 & 87 & BGH 6088 & 61 & BGH 6137 & 91 & BGH 6730 & 90 \\
BGH 5925 & 94 & BGH 6089 & 68 & BGH 6298 & 90 & BGH 7006 & 95 \\
\hline
\end{tabular}

deficientes nesses nutrientes (Wang, 1983). Ainda, por sua rusticidade de cultivo e seu valor nutricional, o taro tem sido sugerido pela FAO (Food and Agriculture Organization), juntamente com outras espécies produtoras de tubérculos e raízes tuberosas, como cultura alternativa para aumentar a base alimentar de países em desenvolvimento (Puiatti, 2001).

No Brasil, com a imigração japonesa, seu valor cultural e comercial tomou grande impulso (Nolasco, 1983). Estima-se que no ano de 1998, o Brasil tenha produzido $225.000 \mathrm{t}$ de rizomas de taro (Camargo Filho et al., 2001). O estado de Minas Gerais, teve em 2001, área plantada de cerca de 990 hectares, com produção de 19.882 t e produtividade de 20,1 $\mathrm{t} \mathrm{ha}^{-1}$ (Puiatti, 2001).

Na família Araceae, à qual pertence o taro, existem centenas de espécies. Algumas possuem resistência ao sombreamento, à seca ou excesso de água, alta capacidade extratora de nutrientes do solo, e algumas apresentam diferentes partes comestíveis (folha, pecíolo e rizomas), características essas que permitem o cultivo das aráceas em diversos ecossistemas. No entanto, o desconhecimento da adaptabilidade das espécies tradicionais aos novos habitats, tem levado ao fracasso inúmeras iniciativas de abertura de novas fronteiras agrícolas (Nolasco, 1983).

Irregularidade do florescimento e anormalidades de estruturas florais têm dificultado o melhoramento de plantas em taro (Ivancic, 1995), fazendo com que seja propagado vegetativamente, razão de se considerar como clones as cultivares de taro já existentes. Apesar de ser espécie com grande plasticidade, podendo adaptar-se a climas desde tropicais úmidos até temperados, sem geada e seco, torna-se necessária a caracterização agronômica desses clones, como forma de se determinar a sua adaptabilidade e estabilidade produtiva.

O presente trabalho teve como objetivo avaliar o rendimento e caracterizar o potencial agronômico de 36 acessos de taro pertencentes ao Banco de Germoplasma de Hortaliças (BGH/ UFV) da UFV.

\section{MATERIAL E MÉTODOS}

O experimento foi conduzido na horta da UFV, de 19/09/2000 a 13/07/ 2001. Utilizou-se o delineamento experimental de blocos casualizados, com 36 tratamentos (acessos ou clones) do $\mathrm{BGH} / \mathrm{UFV}$ e cinco repetições. A parcela foi composta de quatro fileiras espaçadas de 1,0 x 0,5 m, com quatro metros de comprimento, totalizando 32 plantas e a área de $16,0 \mathrm{~m}^{2}$. Foram consideradas área útil as duas fileiras centrais, excluindo-se $0,5 \mathrm{~m}$ das extremidades. As parcelas foram distanciadas de 1,0 $\mathrm{m}$ nas extremidades e de 2,0 $\mathrm{m}$ nas laterais. A área experimental apresenta topografia suave com ligeira inclinação e o solo classificado como Podzólico Vermelho Amarelo Câmbico, fase terraço. Os resultados de análises química e granulométrica de amostras de solo foram: $\mathrm{pH}\left(\mathrm{H}_{2} \mathrm{O}\right)=5,9 ; \mathrm{H}+\mathrm{Al}=2,3, \mathrm{Ca}=$ $1,7 \mathrm{e} \mathrm{Mg}=0,7 \mathrm{cmol} / \mathrm{dm}^{3} ; \mathrm{Na}=4, \mathrm{~K}=$ 125 e $\mathrm{P}=59 \mathrm{mg} / \mathrm{dm}^{3}$; areia grossa $=29$, areia fina $=15$, silte $=17$ e argila $=39$ $\mathrm{dag} / \mathrm{kg}$. O preparo do solo constou de aração e gradagem, sulcamento em linhas, espaçadas de $1,0 \mathrm{~m}$, com profundidade de aproximadamente $12 \mathrm{~cm}$. Utilizou-se no plantio rizomas filhos selecionados por tamanho, com peso médio característico de cada clone, apresentando os pesos médios constantes na Tabela 1. Não foi realizada adubação, no plantio ou em cobertura, e nenhum tipo de controle químico. As irrigações, quando necessárias, foram realizadas semanalmente, por aspersão, e a lâmina de água aplicada, em cada irrigação, foi em média de $40 \mathrm{~mm}$ por semana, considerada satisfatória para atender às necessidades da cultura de taro (Soares, 1991). Foram realizadas, durante o ciclo de cultivo, quatro capinas com auxílio de enxada nas linhas e de cultivador de enxadas tração animal nas entrelinhas, aos 40; 75; 130 e 215 dias após o plantio, respectivamente.

Após nove meses do plantio, atingida a maturação, as plantas foram colhidas. Os rizomas mãe, após separados, e os rizomas filho, após classificados, foram contados e pesados. Os rizomas filho foram classificados, com base no diâmetro transversal, de acordo com Puiatti et al. (1990), nas classes filho grande (>47 mm), filho médio (40-47 $\mathrm{mm})$, filho pequeno $(33-40 \mathrm{~mm})$ e refugo $(<33 \mathrm{~mm})$. Considerou-se como comerciáveis o somatório das classes de rizomas filho grande, médio e pequeno. A produção total consistiu do somatório das produções de rizomas mãe e de todas as classes de rizomas filho. 
Tabela 2. Produção total (PT) e comerciável (PC), peso médio de rizomas comerciáveis (PMRC), número de rizomas comerciáveis por planta (NRCP) e razão de formato de rizomas comerciáveis (RF) em acessos de taro pertencentes ao BGH/UFV. Viçosa, UFV, $2000-2001$.

\begin{tabular}{|c|c|c|c|c|c|}
\hline $\begin{array}{c}\text { Acessos } \\
\text { (BGH) }\end{array}$ & $\begin{array}{c}\text { PT } \\
\text { (t/ha) }\end{array}$ & $\begin{array}{c}P C \\
\text { (t/ha) }\end{array}$ & $\begin{array}{c}\text { PMRC } \\
\text { (g/rizoma) }\end{array}$ & $\begin{array}{c}\text { NRCP } \\
\text { (rizoma/planta) }\end{array}$ & $\begin{array}{c}\text { RF } \\
\text { comp./largura }\end{array}$ \\
\hline BGH 5913 & $27,33 \mathrm{c}$ & $16,27 b$ & $92,08 \mathrm{~b}$ & $8,76 \mathrm{c}$ & $1,72 b$ \\
\hline BGH 5914 & $21,30 \mathrm{c}$ & $15,77 \mathrm{~b}$ & $57,00 \mathrm{~d}$ & $13,74 a$ & $1,58 \mathrm{c}$ \\
\hline BGH 5915 & $28,17 b$ & $16,48 \mathrm{~b}$ & $88,09 \mathrm{~b}$ & $9,30 \mathrm{c}$ & $1,46 \mathrm{c}$ \\
\hline BGH 5916 & $40,15 a$ & $22,45 \mathrm{a}$ & 105,12 a & $10,62 b$ & $1,37 \mathrm{~d}$ \\
\hline BGH 5917 & $30,24 \mathrm{~b}$ & $18,84 \mathrm{a}$ & $88,10 \mathrm{~b}$ & $10,62 b$ & $1,65 b$ \\
\hline BGH 5918 & $22,22 \mathrm{c}$ & $13,78 \mathrm{c}$ & $55,93 \mathrm{~d}$ & $11,80 \mathrm{~b}$ & $1,66 \mathrm{~b}$ \\
\hline BGH 5920 & $19,48 \mathrm{c}$ & $9,35 \mathrm{c}$ & $61,07 \mathrm{c}$ & $7,66 \mathrm{~d}$ & $1,55 \mathrm{c}$ \\
\hline BGH 5921 & $21,89 \mathrm{c}$ & $15,55 \mathrm{~b}$ & $56,35 d$ & 13,38 a & $1,48 \mathrm{c}$ \\
\hline BGH 5925 & $36,30 \mathrm{a}$ & $22,54 \mathrm{a}$ & 91,32 b & $12,36 \mathrm{~b}$ & $1,30 \mathrm{~d}$ \\
\hline BGH 5926 & 17,22 c & $9,29 \mathrm{c}$ & $49,29 d$ & $8,90 \mathrm{c}$ & $1,49 c$ \\
\hline BGH 5927 & $24,30 \mathrm{c}$ & $9,94 \mathrm{c}$ & $94,17 \mathrm{~b}$ & $5,30 \mathrm{~d}$ & $1,34 \mathrm{~d}$ \\
\hline BGH 5928 & $19,84 \mathrm{c}$ & $13,90 \mathrm{c}$ & $52,99 \mathrm{~d}$ & $13,02 \mathrm{a}$ & $1,72 b$ \\
\hline BGH 5929 & $19,98 \mathrm{c}$ & $10,66 \mathrm{c}$ & $62,87 \mathrm{c}$ & $8,46 \mathrm{c}$ & $1,14 \mathrm{e}$ \\
\hline BGH 5931 & 19,02 c & $9,38 \mathrm{c}$ & $67,89 \mathrm{c}$ & $6,74 \mathrm{~d}$ & $1,68 \mathrm{~b}$ \\
\hline BGH 6086 & $23,65 \mathrm{c}$ & $15,40 \mathrm{~b}$ & $47,20 \mathrm{~d}$ & $16,00 \mathrm{a}$ & $1,69 \mathrm{~b}$ \\
\hline BGH 6087 & $28,92 \mathrm{~b}$ & $16,07 \mathrm{~b}$ & $76,80 \mathrm{c}$ & $10,40 \mathrm{~b}$ & $1,39 d$ \\
\hline BGH 6088 & $21,26 \mathrm{c}$ & $14,50 \mathrm{~b}$ & $56,16 \mathrm{~d}$ & $13,00 \mathrm{a}$ & $1,62 b$ \\
\hline BGH 6089 & $16,58 \mathrm{c}$ & $8,80 \mathrm{c}$ & $60,81 \mathrm{c}$ & $6,88 \mathrm{~d}$ & $0,96 \mathrm{e}$ \\
\hline BGH 6091 & $22,36 \mathrm{c}$ & $11,44 \mathrm{c}$ & 63,07 c & $8,92 \mathrm{c}$ & $1,30 \mathrm{~d}$ \\
\hline BGH 6092 & $14,02 \mathrm{c}$ & $6,44 \mathrm{c}$ & $54,98 \mathrm{~d}$ & $5,56 \mathrm{~d}$ & $0,96 \mathrm{e}$ \\
\hline BGH 6093 & $27,56 \mathrm{~b}$ & $16,34 b$ & $74,11 \mathrm{c}$ & $10,92 b$ & $1,46 \mathrm{c}$ \\
\hline BGH 6094 & $22,20 \mathrm{c}$ & $12,75 \mathrm{c}$ & $70,67 c$ & $8,86 \mathrm{c}$ & $1,40 \mathrm{~d}$ \\
\hline BGH 6095 & $21,35 \mathrm{c}$ & $11,90 \mathrm{c}$ & $70,65 \mathrm{c}$ & $8,50 \mathrm{c}$ & $1,25 d$ \\
\hline BGH 6132 & $16,96 \mathrm{c}$ & $8,70 \mathrm{c}$ & $64,52 \mathrm{c}$ & $6,48 \mathrm{~d}$ & $1,02 \mathrm{e}$ \\
\hline BGH 6136 & $22,61 \mathrm{C}$ & $15,18 b$ & $66,02 \mathrm{c}$ & $11,32 \mathrm{~b}$ & $1,62 b$ \\
\hline BGH 6137 & $36,53 \mathrm{a}$ & 22,36 a & $110,61 \mathrm{a}$ & $9,88 \mathrm{c}$ & $1,31 \mathrm{~d}$ \\
\hline BGH 6298 & $36,74 \mathrm{a}$ & $21,76 \mathrm{a}$ & $110,62 \mathrm{a}$ & $9,78 \mathrm{c}$ & $1,62 b$ \\
\hline BGH 6306 & $18,50 \mathrm{c}$ & $9,57 \mathrm{c}$ & $63,97 \mathrm{c}$ & $7,54 \mathrm{~d}$ & $1,14 \mathrm{e}$ \\
\hline BGH 6307 & $30,13 \mathrm{~b}$ & 20,72 a & $63,72 \mathrm{c}$ & $16,22 \mathrm{a}$ & $1,61 \mathrm{~b}$ \\
\hline BGH 6308 & $27,34 \mathrm{~b}$ & $19,32 \mathrm{a}$ & $63,40 \mathrm{c}$ & $15,14 a$ & $1,74 \mathrm{~b}$ \\
\hline BGH 6315 & $27,66 \mathrm{~b}$ & 17,46 b & $64,23 \mathrm{c}$ & $13,56 a$ & $2,09 a$ \\
\hline BGH 6606 & $23,68 \mathrm{c}$ & $13,53 \mathrm{c}$ & $64,93 \mathrm{c}$ & $10,34 \mathrm{~b}$ & $1,18 \mathrm{e}$ \\
\hline BGH 6607 & $15,40 \mathrm{c}$ & $10,49 \mathrm{c}$ & $49,17 d$ & $10,58 \mathrm{~b}$ & $1,78 \mathrm{~b}$ \\
\hline BGH 6708 & $21,96 \mathrm{c}$ & $15,40 \mathrm{~b}$ & $58,57 \mathrm{~d}$ & 13,08 a & $1,11 \mathrm{e}$ \\
\hline BGH 6730 & $28,58 \mathrm{~b}$ & $18,14 b$ & $77,29 \mathrm{c}$ & $11,68 \mathrm{~b}$ & $1,73 \mathrm{~b}$ \\
\hline BGH 7006 & $30,88 \mathrm{~b}$ & $17,94 \mathrm{~b}$ & $95,51 \mathrm{~b}$ & $9,50 \mathrm{c}$ & $1,61 \mathrm{~b}$ \\
\hline Média geral & 24,51 & 14,67 & 70,81 & 10,41 & 1,46 \\
\hline CV (\%) & 27,12 & 29,28 & 17,58 & 9,61 & 10,72 \\
\hline
\end{tabular}

Médias seguidas pela mesma letra na coluna não diferem entre si, ao nível de 5\% de probabilidade, pelo teste de Scott Knott.

Avaliou-se a produtividade de rizomas comerciáveis e total; peso médio de rizomas comerciáveis; número de rizomas comerciáveis por planta; razão de forma- to (diâmetro longitudinal/diâmetro transversal) de rizomas comerciáveis; produtividade de rizomas em classes (com base no diâmetro transversal) filho grande, médio, pequeno e refugo. Quanto à razão de formato considerou-se como rizomas esféricos até 1,0, oblongos de 1,1 a 1,7 e cilíndricos acima de 1,7. 
As análises estatísticas foram realizadas utilizando-se o programa SAEG (Sistema de Análises Estatísticas e Genéticas da UFV) e, às médias, aplicado o teste de Scott Knott a 5\% de probabilidade. Os dados de número de rizomas comerciáveis por planta foram transformados em raiz quadrada de $(x+1)$, para realização das análises. Correlações de Pearson foram realizadas entre as características agronômicas.

\section{RESULTADOS E DISCUSSÃO}

Para todas as características avaliadas houve efeito significativo de acessos $(\mathrm{P}<0,05)$. Os resultados médios dos clones, quanto às características estudadas, encontram-se nas Tabelas 2, 3 e 4.

As produtividades totais e comerciáveis de rizomas foram dispostas em três grupos; os pesos médios e números por planta de rizomas comerciáveis em quatro grupos e as razões de formato em cinco grupos, estatisticamente distintos. A produtividade total média de rizomas, dos 36 acessos, foi de 24,5 t/ha, com valores variando, dentro de cada grupo, de 40,2 a 36,3 ; 30,9 a 27,3 e de 27,3 a 14,0 t/ha, respectivamente. A produtividade média de rizomas comerciáveis, dos 36 acessos, foi de 14,7 t/ha, com valores variando, em cada grupo, de 22,5 a 18,8; 17,2 a 14,5 e de 13,9 a 6,4 t/ha, respectivamente. O peso médio de rizomas comerciáveis, dos 36 acessos, foi de 70,8 $\mathrm{g}$, com valores variando, em cada grupo, de 110,6 a 105,$1 ; 95,5$ a 88,1; 77,3 a 60,8 e de 59,0 a 47,2 g/rizoma, respectivamente. O número de rizomas comerciáveis por planta, dos 36 acessos, foi de 10,4, com valores variando, dentro de cada grupo, de 16,2 a 13,0; 12,4 a 10,$3 ; 9,9$ a 8,5 e de 7,7 a 5,3 , respectivamente. A razão de formato de rizomas comerciáveis, dos 36 acessos, foi de 1,5 , com valores variando, dentro de cada grupo, de 2,09 a 1,77; 1,78 a 1,$61 ; 1,58$ a 1,$46 ; 1,40$ a 1,25 e de 1,18 a 0,96 , respectivamente.

Os clones BGH 5916, BGH 6298, BGH 6137 e Japonês-BGH 5925 apresentaram maiores produtividades totais (Tabela 2). As produtividades alcançadas por estes clones estão relacionadas ao equilíbrio entre a produti- vidade de rizomas comerciáveis, representado principalmente pela produtividade de rizomas filho grande, e a produção de rizomas mãe (Tabelas 2 e 3). No clone Chinês-BGH 5928, apesar do número de rizomas comerciáveis por planta ser significativamente maior que o apresentado pelos clones com maior produtividade total, a produtividade de rizomas comerciáveis e rizomas mãe foi menor, não correspondendo portanto, em termos de produção total de rizomas (Tabelas 2 e 3). A produção do clone Japonês-BGH 5925 não diferiu dos clones que apresentaram maior produção total, indicando ser realmente um material bastante produtivo.

A produção de rizomas mãe e filhos grandes apresentam os maiores efeitos sobre o rendimento total de rizomas e pode, portanto, ser usada como critério de seleção para alto rendimento em genótipos de taro, apesar de, no momento, a produção elevada de rizomas mãe não ser de interesse econômico. Quanto à produtividade total, resultados semelhantes aos obtidos nesse trabalho foram encontrados por Carmo \& Ferrão (2000) em solo fertilizado, com valores variando entre $27,1 \mathrm{t} /$ ha no clone Cem/ um-BGH 6086 a 48,3 t/ha no clone Chinês-R. Heredia Zárate et al. (2000) também obtiveram produtividade total expressiva com adição de cama de frango de corte semidecomposta nos clones Macaquinho $(68,6)$, Branco $(64,7)$, Cem/um-BGH6086 (59,8), JaponêsBGH $5925(54,4)$ e Chinês-BGH 5928 $(49,1) \mathrm{t} / \mathrm{ha}$. No entanto, resultados menos expressivos de produtividade total foram obtidos por Heredia Zárate (1990) nos clones Macaquinho (16,0), Roxo $(12,9)$, Chinês-BGH $5928(9,9)$ e No 5 $(9,8) \mathrm{t} / \mathrm{ha}$.

Quando comparadas às produtividades de cultivares comerciais de taro de outros países, os clones aqui avaliados foram promissores. Pandey \& Dobhal (1997) obtiveram produtividade total, em clones indianos, variando de 8,5 a 20,8 t/ha. Goenaga \& Chardon (1995), obtiveram, com cvs. Porto Riquenhas, produtividade total média de 20,2 t/ha. Lehua, uma das mais importantes variedades do Havaí, apresentou produtividade total variando de 10,0 a 57,6 t/ha, dependendo das doses de adubação nitrogenada e épocas de colheita (Plucknett \& de la Peña, 1971). Liou (1984) obteve, com a cv. chinesa Betelnut, produtividade total de 25,8 a $63,3 \mathrm{t} / \mathrm{ha}$, dependendo da densidade de plantio utilizada, mantendo fixo o espaçamento de $60 \mathrm{~cm}$ entre fileiras, com variações no espaçamento entre plantas de 20 a $60 \mathrm{~cm}$.

Os clones Japonês-BGH 5925, BGH 5916, BGH 5917, BGH 6137, BGH 6298, BGH 6307 e BGH 6308 apresentaram maior produção de rizomas comerciáveis (Tabela 2). A maior produção de rizomas comerciáveis alcançada pelos clones Japonês-BGH 5925, BGH 5916 e BGH 5917, está relacionada ao equilíbrio entre número de rizomas comerciáveis por planta e seu peso médio, características essas correlacionadas positiva e significativamente com a produtividade de rizomas comerciáveis, com valores de 0,7133 e 0,6370 , respectivamente (Tabela 4 ), sendo portanto uma característica desejável quando o objetivo é atingir altas produtividades. Uma das características que pode também ser considerada relevante para o bom desempenho desses clones, diz respeito à alta produtividade de rizomas filho grande e baixa produtividade nas demais classes (Tabela 3), com exceção dos clones BGH 5917, BGH 6307 e BGH 6308. Essa afirmativa é reforçada pela alta correlação positiva e significativa $(0,9107)$ obtida entre a produtividade de rizomas comerciáveis e a produtividade de rizomas filho grande (Tabela 4).

Nos clones BGH 6137 e BGH 6298 o fator determinante na obtenção de alta produção de rizomas comerciáveis foi o maior peso médio de rizomas comerciáveis, enquanto que nos clones BGH 6307 e BGH 6308 foi o maior número de rizomas comerciáveis por planta (Tabela 2). No clone ChinêsBGH 5928 apesar de o número de rizomas comerciáveis por planta ter sido superior ao clone Japonês-BGH 5925, seu peso médio foi bem menor, resultando em menor produtividade de rizomas comerciáveis (Tabela 2). Resultados semelhantes foram encontrados por Carmo \& Ferrão (2000), em solo fertilizado, com valores que variaram de 17,3 t/ha para o clone Cem/um-BGH 
Tabela 3. Produção de rizomas mãe (PRM), filho grande (PFG), filho médio (PFM), filho pequeno (PFP), refugo (PREF) em acessos de taro pertencentes ao BGH/UFV. Viçosa, UFV, 2000-2001.

\begin{tabular}{|c|c|c|c|c|c|}
\hline $\begin{array}{r}\text { Acessos } \\
\text { (BGH) }\end{array}$ & $\begin{array}{l}\text { PRM } \\
\text { (t/ha) }\end{array}$ & $\begin{array}{l}\text { PFG } \\
\text { (t/ha) }\end{array}$ & $\begin{array}{l}\text { PFM } \\
\text { (t/ha) }\end{array}$ & $\begin{array}{l}\text { PFP } \\
\text { (t/ha) }\end{array}$ & $\begin{array}{l}\text { PREF } \\
\text { (t/ha) }\end{array}$ \\
\hline BGH 5913 & $10,79 b$ & $13,12 b$ & $2,42 \mathrm{c}$ & $0,73 d$ & $0,27 \mathrm{~d}$ \\
\hline BGH 5914 & $5,02 \mathrm{c}$ & $7,81 \mathrm{c}$ & $5,50 a$ & $2,47 \mathrm{~b}$ & $0,51 \mathrm{~d}$ \\
\hline BGH 5915 & 11,42 b & 13,07 b & $2,52 \mathrm{c}$ & $0,99 d$ & $0,27 d$ \\
\hline BGH 5916 & $17,32 \mathrm{a}$ & 19,76 a & $1,86 \mathrm{~d}$ & $0,82 \mathrm{~d}$ & $0,39 d$ \\
\hline BGH 5917 & $11,00 \mathrm{~b}$ & 15,04 b & $2,52 \mathrm{c}$ & $1,28 d$ & $0,40 d$ \\
\hline BGH 5918 & $6,67 \mathrm{c}$ & $6,97 \mathrm{c}$ & $4,08 \mathrm{~b}$ & $2,73 b$ & $1,78 \mathrm{~b}$ \\
\hline BGH 5920 & $9,41 \mathrm{c}$ & $5,07 \mathrm{c}$ & $2,55 \mathrm{c}$ & $1,74 \mathrm{c}$ & $0,72 \mathrm{c}$ \\
\hline BGH 5921 & $5,69 \mathrm{c}$ & $8,70 \mathrm{c}$ & $4,35 \mathrm{~b}$ & $2,51 \mathrm{~b}$ & $0,64 d$ \\
\hline BGH 5925 & $13,23 a$ & 18,32 a & $2,87 \mathrm{c}$ & $1,35 \mathrm{c}$ & $0,52 d$ \\
\hline BGH 5926 & $6,97 \mathrm{c}$ & $3,94 \mathrm{c}$ & $2,74 \mathrm{c}$ & $2,52 \mathrm{~b}$ & $1,06 \mathrm{c}$ \\
\hline BGH 5927 & $13,44 \mathrm{a}$ & $7,86 \mathrm{c}$ & $0,89 \mathrm{~d}$ & $1,19 d$ & $0,91 \mathrm{c}$ \\
\hline BGH 5928 & $4,82 \mathrm{c}$ & $6,05 \mathrm{c}$ & $5,09 a$ & $2,76 \mathrm{~b}$ & $1,13 \mathrm{c}$ \\
\hline BGH 5929 & $7,09 \mathrm{c}$ & $6,53 \mathrm{c}$ & $1,67 d$ & $2,47 \mathrm{~b}$ & $2,22 \mathrm{a}$ \\
\hline BGH 5931 & $8,56 \mathrm{c}$ & $5,93 \mathrm{c}$ & $1,86 \mathrm{~d}$ & $1,58 \mathrm{c}$ & $1,08 \mathrm{c}$ \\
\hline BGH 6086 & $5,58 \mathrm{c}$ & $5,23 \mathrm{c}$ & 4,83 a & $5,34 a$ & $2,67 \mathrm{a}$ \\
\hline BGH 6087 & $11,38 \mathrm{~b}$ & $11,18 \mathrm{c}$ & $2,66 \mathrm{c}$ & $2,24 \mathrm{~b}$ & $1,46 \mathrm{~b}$ \\
\hline BGH 6088 & $5,57 \mathrm{c}$ & $7,02 \mathrm{c}$ & 4,99 a & $2,50 \mathrm{~b}$ & $1,18 \mathrm{c}$ \\
\hline BGH 6089 & $6,19 c$ & $5,73 \mathrm{c}$ & $1,03 d$ & $2,04 \mathrm{c}$ & $1,59 \mathrm{~b}$ \\
\hline BGH 6091 & 10,05 b & $7,34 \mathrm{c}$ & $2,24 \mathrm{c}$ & $1,86 \mathrm{c}$ & $0,87 \mathrm{c}$ \\
\hline BGH 6092 & $6,05 \mathrm{c}$ & $3,94 \mathrm{c}$ & $1,08 d$ & $1,43 \mathrm{c}$ & $1,53 \mathrm{~b}$ \\
\hline BGH 6093 & $9,54 \mathrm{c}$ & $1,05 \mathrm{c}$ & $2,68 \mathrm{c}$ & $3,13 b$ & $1,68 \mathrm{~b}$ \\
\hline BGH 6094 & $8,33 \mathrm{c}$ & $8,06 \mathrm{c}$ & $2,40 \mathrm{c}$ & $2,29 \mathrm{~b}$ & $1,13 \mathrm{c}$ \\
\hline BGH 6095 & $6,97 \mathrm{c}$ & $8,54 \mathrm{c}$ & $1,42 d$ & $1,93 \mathrm{c}$ & $2,48 a$ \\
\hline BGH 6132 & $6,69 \mathrm{c}$ & $5,82 \mathrm{c}$ & $1,09 \mathrm{~d}$ & $1,79 \mathrm{c}$ & $1,57 \mathrm{~b}$ \\
\hline BGH 6136 & $6,64 \mathrm{c}$ & $8,84 \mathrm{c}$ & 4,26 b & $2,08 \mathrm{C}$ & $0,79 \mathrm{c}$ \\
\hline BGH 6137 & $14,10 \mathrm{a}$ & 19,33 a & $2,31 \mathrm{c}$ & $0,62 \mathrm{~d}$ & $0,17 \mathrm{~d}$ \\
\hline BGH 6298 & $14,72 \mathrm{a}$ & 18,79 a & $2,17 \mathrm{c}$ & $0,80 \mathrm{~d}$ & $0,26 \mathrm{~d}$ \\
\hline BGH 6306 & $7,24 \mathrm{c}$ & $5,82 \mathrm{c}$ & $1,57 \mathrm{~d}$ & $2,18 b$ & $1,69 \mathrm{~b}$ \\
\hline BGH 6307 & $8,20 \mathrm{c}$ & $11,28 \mathrm{c}$ & $6,30 \mathrm{a}$ & $3,14 \mathrm{~b}$ & $1,21 \mathrm{c}$ \\
\hline BGH 6308 & $6,96 \mathrm{c}$ & $11,08 \mathrm{c}$ & $5,30 \mathrm{a}$ & $2,95 \mathrm{~b}$ & $1,06 \mathrm{c}$ \\
\hline BGH 6315 & $7,54 \mathrm{c}$ & $7,40 \mathrm{c}$ & $5,05 a$ & $5,02 \mathrm{a}$ & $2,66 \mathrm{a}$ \\
\hline BGH 6606 & $8,74 \mathrm{c}$ & $7,97 \mathrm{c}$ & $3,07 \mathrm{c}$ & $2,49 \mathrm{~b}$ & $1,31 \mathrm{c}$ \\
\hline BGH 6607 & $3,78 \mathrm{c}$ & $3,50 \mathrm{c}$ & $4,21 \mathrm{~b}$ & $2,78 \mathrm{~b}$ & $1,13 \mathrm{c}$ \\
\hline BGH 6708 & $5,64 \mathrm{c}$ & $7,50 \mathrm{c}$ & $5,42 a$ & $2,48 \mathrm{~b}$ & $0,92 \mathrm{c}$ \\
\hline BGH 6730 & 9,96 b & $12,76 \mathrm{~b}$ & 3,91 b & $1,47 \mathrm{c}$ & $0,47 \mathrm{~d}$ \\
\hline BGH 7006 & $12,64 \mathrm{~b}$ & $14,60 \mathrm{~b}$ & $2,49 \mathrm{c}$ & $0,85 d$ & $0,29 d$ \\
\hline Média geral & 8,72 & 9,46 & 3,09 & 2,12 & 1,11 \\
\hline CV (\%) & 31,84 & 42,89 & 31,28 &, 06 & 46,05 \\
\hline
\end{tabular}

Médias seguidas pela mesma letra na coluna não diferem entre si, ao nível de 5\% de probabilidade, pelo teste de Scott Knott.

6086 a 33,8 t/ha para o clone Macaquinho. Katsumoto \& Puiatti (1990) obtiveram, com o clone Chinês-BGH 5928, produtividades de rizomas comerciáveis que variaram de 10,2 a 25,8 t/ha, dependendo do tipo de muda utilizada, em que os tipos cabeça central intacta, rizomas filho grande, filho médio e filho pequeno, propiciaram produtividades comerciáveis de 25,$8 ; 23,7 ; 22,5$ e $18,5 \mathrm{t} / \mathrm{ha}$, respectivamente. 
Tabela 4. Correlação fenotípica em acessos de taro pertencentes ao BGH/UFV. Viçosa, UFV, 2000-2001.

\begin{tabular}{|c|c|c|c|c|c|c|}
\hline Variáveis & PT & PC & PMRC & NRCP & PFG & PRM \\
\hline PT & $1,0000^{* *}$ & $0,9455^{* *}$ & 0,7450 ** & $0,5338^{* *}$ & $0,9451^{* *}$ & $0,8753^{* *}$ \\
\hline PC & & $1,0000^{* *}$ & $0,6370^{* *}$ & $0,7133^{* *}$ & $0,9107^{* *}$ & ns \\
\hline PMRC & & & $1,0000^{* *}$ & ns & $0,8519 * *$ & ns \\
\hline NRCP & & & & $1,0000^{* *}$ & $0,3814^{* *}$ & ns \\
\hline PFG & & & & & $1,0000^{* *}$ & ns \\
\hline PRM & & & & & & $1,000 * *$ \\
\hline
\end{tabular}

1/Peso médio de rizomas comerciais (PMRC); número de rizomas comerciais por planta (NRCP); produtividades de rizomas mãe (PRM), filho grande (PFG), comerciáveis (PC) e total (PT).

$2 / * *$; ns, significativo a $1 \%$ e não significativo, respectivamente.

A produtividade de rizomas comerciáveis é o objetivo principal de uma exploração comercial de taro. No entanto, verifica-se que existe variação bastante ampla, entre os trabalhos citados, quanto a produtividade de um mesmo clone para os diferentes locais e épocas de cultivo, evidenciando existir, além de diferentes práticas de manejo adotadas, interação genótipo x ambiente bastante acentuada.

Os clones Japonês-BGH 5925 e Chinês-BGH 5928, os mais cultivados comercialmente, apresentaram pesos médios de rizomas comerciáveis de 91,3 e $53,0 \mathrm{~g}$, respectivamente. Estes resultados, principalmente do BGH 5928, estão aquém dos encontrados nos clones BGH 5916, BGH 6137 e BGH 6298 (Tabela 2). O maior peso médio de rizomas comerciáveis dos clones $\mathrm{BGH}$ 5916, BGH 6137 e BGH 6298, em relação ao Chinês-BGH 5928, pode ser atribuído à presença de menor número de rizomas comerciáveis por planta, o que favorece o acúmulo de reservas nesses rizomas, demonstrado pela maior produção de rizomas filho grande (Tabela 2). No clone Japonês-BGH 5925 a diferença no peso médio dos rizomas com relação aos clones BGH 5916, BGH 6137 e BGH 6298, não foi suficientemente grande que pudesse vir a interferir na produção de rizomas comerciáveis e totais, pois a produção de rizomas filho grande e médio, com exceção do clone BGH 5916, não apresentaram diferenças significativas. Estes resultados foram bastante expressivos quando comparados a 23,9 g/rizoma no clone Chinês-BGH 5928 encontrados por Nolasco (1984) e nos clones JaponêsBGH 5925 (28,7), Macaquinho $(27,1)$,
Chinês-BGH $5928(24,4)$, Cem/umBGH $6086(14,5)$ e Branco $(11,9) \mathrm{g} /$ rizoma, encontrados por Heredia Zárate (1995). Todavia, a falta de registro dos códigos de muitos desses acessos trabalhados por esses autores, dificultam comentários comparativos. O maior peso médio dos rizomas aqui encontrados, para os mesmos clones, pode estar relacionado com o maior espaçamento usado nesse experimento, além da não distinção entre rizomas filhos comerciáveis e refugo pelos autores citados.

$\mathrm{O}$ peso médio de rizomas comerciáveis é de grande importância para tomada de decisão por parte do agricultor, haja vista que, dependendo do mercado e da variação de preços de rizomas comerciais, os de maior peso médio (filho grande) alcançam preços 20 a $30 \%$ superiores aos rizomas médios e até $80 \%$ acima dos rizomas pequenos, o que pode ser uma estratégia para se conseguir maior lucratividade.

Os clones BGH 6307, BGH 6086, BGH 6308, BGH 5914, BGH 6315, BGH 6708, BGH 5921, Chinês-BGH 5928 e BGH 6088 apresentaram maior número de rizomas comerciáveis por planta (Tabela 2). Apesar da correlação positiva entre o número de rizomas comerciáveis por planta com a produtividade de rizomas comerciáveis (Tabela 4), com exceção dos clones BGH 6307 e BGH 6308, esse número não foi suficientemente alto para compensar o baixo peso médio de seus rizomas e conseqüentemente elevar as produtividades de rizomas comerciáveis desses clones. Isso indica que a produção de rizomas comerciáveis/área não é diretamente proporcional ao número de rizomas comerciáveis por planta não sendo, portanto, uma característica indicada para previsão do rendimento comercial. Diferentemente, Pillai et al. (1995) encontraram que, entre as características agronômicas, o número de rizomas filho por planta foi o que exerceu maior influência sobre o rendimento, seguido pelo peso médio.

Os clones Japonês-BGH 5925 e Chinês-BGH 5928 apresentaram número médio de rizomas comerciáveis por planta de 12,4 e 13,0, respectivamente, sendo estes valores estatisticamente distintos (Tabela 2), conforme exposto por Puiatti (2001), o qual afirma que o clone Chinês-BGH 5928 apresenta maior número de rizomas/planta. Heredia Zárate (1995) obteve valores que variaram de 8,0 rizomas/planta no clone Chinês-BGH 5928 a 33 rizomas/planta no clone Branco, seguido do Macaquinho com 31 rizomas por planta. No entanto, em razão do baixo peso médio dos rizomas encontrados nesses trabalhos acredita-se que não foi feita distinção entre rizomas comerciáveis e refugo, além da falta de registro dos códigos dos acessos trabalhados por esses autores, dificultando a exposição de comentários comparativos.

A grande amplitude verificada, quanto ao número de rizomas comerciáveis por planta, entre os clones aqui avaliados, sugere a existência de ampla variabilidade genética, que poderia ser utilizada em programas de melhoramento, apesar do pouco sucesso obtido nessa prática, até então, devido às dificuldades dessa espécie, em se reproduzir sexuadamente (Ivancic, 1995).

Os clones BGH 6089 e BGH 6092, ambos com razão de formato de 1,0, são 
esféricos. Os clones BGH 6315, BGH 6607, BGH 6308, BGH 6730, BGH 5913 e BGH 5928 com razão de formato acima de 1,7 , apresentaram formato cilíndrico. Os demais clones, com razão de formato variando de 1,1 a 1,7 , apresentaram formato oblongo (Tabela 2).

Os clones Japonês-BGH 5925 e Chinês-BGH 5928 apresentaram razão de formato para os rizomas comerciáveis de 1,3 (oblongo) e 1,7 (cilíndrico), respectivamente. Esses resultados comprovam os relatados por Puiatti (2001), de que o clone Chinês-BGH 5928 apresenta formato de rizomas mais alongado e o Japonês-BGH 5925 formato mais ovalado. A razão de formato é uma característica comercial muito importante, pois existe preferência diferenciada entre os consumidores tradicionais que preferem rizomas mais alongados e de tamanho médio, enquanto os não tradicionais preferem rizomas mais ovalado e de tamanho grande. Futuramente, do ponto de vista industrial, a razão de formato poderá ser importante para determinar qual ou quais os tipos de rizomas seriam mais apropriados para um possível descasque mecânico, com vista ao seu beneficiamento.

Tendo em vista os resultados obtidos nesse trabalho, fica evidente o bom desempenho de clones, até então desconhecidos, tais com BGH 5916, BGH 6298 e BGH 6137, apresentando as melhores respostas agronômicas quanto a peso médio de rizomas comerciáveis, produtividades de rizomas comerciáveis e total, que não diferem do clone Japonês-BGH 5925 e superando o clone Chinês-BGH 5928. Esses novos clones apresentam rizomas comerciáveis com peso médio maior e boa conformação, com razão de formato entre 1,1 e 1,7 (rizomas oblongos), características essas que lhes garantem melhor cotação em mercados mais exigentes quanto ao aspecto do produto, porém menos tradicionais no consumo de taro (Puiatti, 2001).
Os clones BGH 6307 e BGH 6308, também obtiveram bons resultados, com peso médio de rizomas comerciais não tão elevados quanto os clones BGH 6298, BGH 6137 e BGH 5916, no entanto com número de rizomas comerciáveis por planta elevado, propiciaram elevada produtividade de rizomas comerciáveis, não diferindo dos anteriores, e com produtividade total razoável. Em decorrência da grande mudança de hábito do consumidor nos últimos anos, não somente no Brasil, é possível que rizomas com peso médio menores, como o desses clones, bem como do clone Cem/um-BGH 6086, possam vir a ter grande valor de comercialização. É importante ressaltar que apesar de os clones BGH 5916, BGH 6137, BGH 6298, BGH 6307 e BGH 6308 serem promissores, é necessário testar esses materiais em outros ambientes.

\section{LITERATURA CITADA}

CAMARGO FILHO, W.P.; MAZZEI, A.R.; ALVES, H.S. Mercado de raízes e tubérculos: análise de preços. Informações Econômicas, São Paulo, v. 31, n. 2, p. 36-44, fev. 2001.

CARMO, C.A.S.; FERRÃO, M.A.G. Comportamento de clones de inhame na região centro - serrana do Estado do Espírito Santo. Horticultura Brasileira, Brasília, v. 18, p. 591-593, Suplemento, julho 2000.

GOENAGA, R.; CHARDON, U. Growth, yield and nutrient uptake of taro grown under upland conditions. Journal of Plant Nutrition, v. 18, n. 5 , p. 1037-1048, 1995.

HEREDIA ZÁRATE, N.A. Curvas de crescimento de quarto cultivares de inhame, considerando quatro tipos de mudas e três populações. In: ENCONTRO NACIONAL SOBRE A CULTURA DO INHAME, II. 1989, Dourados, Anais... Campo Grande: UFMS, 1990. p. 59-70.

HEREDIA ZÁRATE, N.A. Produção de clones de inhame cultivados no pantanal sul matogrossense. Horticultura Brasileira, Brasília, v. 13, n. 1, p. 38-40, 1995.

HEREDIA ZÁRATE, N.A.; VIEIRA, M.C.; ROSA Jr., E.J.; SILVA, C.G. Formas de adição ao solo de cama-de-frangos de corte semidecomposta para a produção de inhame. Horticultura Brasileira, Brasília, v. 18, p. 521-523, Suplemento, julho 2000.
IVANCIC, A. Abnormal and unusual inflorescences of taro, Colocasia esculenta (Araceae). Australian Journal Botany, v. 43, p. 475-489, 1995.

KATSUMOTO, R.; PUIATTI, M. Utilização da cabeça central como rizoma-muda na cultura do inhame (Colocasia esculenta) 'Chinês'. In: RESUMOS DO CONGRESSO BRASILEIRO DE OLERICULTURA, XXX, Campo Grande, MS. 1990. Horticultura Brasileira, Brasília, v. 8, n. 1, p. $47,1990$.

LIOU, T.D. Effects of plant density on the yield of taro in paddy field. Journal Agricultural Research of China, v. 33, n. 1, p. 38-43, 1984.

NOLASCO, F. Estudos para o cultivo inundado do inhame (Colocasia esculenta (L.) Schott), em monocultivo e em consórcio com azolla. Viçosa: UFV, 1984. 80 p. (Tese mestrado).

NIP, WAI - KIT. Taro food products. In: HOLLYER, J.R. \& SATO, D.M., ed. Taking taro into the 1990: a taro conference. Proceedings... Hawaii: University of Hawaii, 1990. p. 3-5.

NOLASCO, F. Aspectos gerais da cultura do inhame [Colocasia esculenta (L.) Schoot]. In: HEREDIA, M.S., BURBA, J.L., CASALI, V.W.D. (Coord.). Seminários de Olericultura. Viçosa: UFV, 1983. v. 6, p. 21-36.

PANDEY, G.; DOBHAL, V.K. Multivariate analysis in taro (Colocasia esculenta (L.) Schott). Indian Journal of Genetics and Plant Breeding, v. 57, n. 3, p. 262-265, 1997.

PILLAI, P.K.T; LAKSHMI, K.R.; SHEELA, M.N. Correlation and path analysis in taro. Journal of Root Crops, v. 21, n. 2, p. 86-89, 1995.

PLUCKNETT, D.L.; PEÑA, R.S. Taro production in Hawai, World Crops, v. 23, n. 5, p. 244-249, 1971.

PUIATTI, M. Curso técnico sobre a cultura do taro (Colocasia esculenta (L.) Schott). In: CARMO, C.A.S. Coord. I Simpósio Nacional Sobre as Culturas do Inhame e do Cará. Venda Nova do Imigrante: Incaper, 2001. p. 1-44.

PUIATTI, M.; CAMPOS, J. P.; CASALI, V.W.D.; CARDOSO, A.A.; CRUZ, R. Sistema de colocação do bagaço de cana-de-açúcar e capim gordura, na cultivar de inhame Chinês. Horticultura Brasileira, Brasília, v. 8, n. 1, p. 14-16, 1990.

SOARES, J.G. Crescimento do inhame (Colocasia esculenta (L.) Schott) em duas condições agroclimáticas, em seis níveis de água e cobertura morta. Viçosa: UFV, 1991. 91 p. (Tese mestrado).

STANDAL, B.R. Nutritive Value. In: WANG, J.K., HIGA, S. ed. Taro: A review of Colocasia esculenta and its potentials. Honolulu: University of Hawaii Press, 1983. p. 141-147.

WANG, J.K. Introduction. In: WANG, J.K., HIGA, S. ed. Taro: A review of Colocasia esculenta and its potentials. Honolulu: University of Hawaii Press, 1983. p. 3-13. 